\title{
Increase in Grasp Force Reflects a Desire to Improve Movement Precision
}

\author{
(1) A. Takagi, ${ }^{1,2}$ H. Kambara, ${ }^{1}$ and Y. Koike ${ }^{1}$
}

https://doi.org/10.1523/ENEURO.0095-19.2019

\begin{abstract}
${ }^{1}$ Tokyo Institute of Technology, Institute of Innovative Research, Yokohama 226-8503, Japan and ${ }^{2}$ Precursory Research for Embryonic Science and Technology (PRESTO), Japan Science and Technology Agency (JST), Kawaguchi, Saitama 332-0012, Japan
\end{abstract}

\begin{abstract}
Grasping is an action engraved in the human genome, enabling newborn infants to hang from a monkey-bar immediately after birth. The grasp force provides rich information about the brain's control of arm movements. In this study, we tested the hypothesis that the grasp force increases to improve the hand's movement precision during reaching. In two reaching experiments, subjects increased grasp force to suppress movement imprecision that arose from both self-generated motor noise and from an unpredictable environment. Furthermore, the grasp force did not increase constantly, but increased specifically along the movement where the hand's deviation was greatest. The increased grasp was premeditated and was not a reaction to environmental forces, suggesting that the central nervous system has a predictive, state-dependent model of movement precision during reaching. The grasp force provides a high temporal resolution and calibration-less estimate of movement precision adaptation.
\end{abstract}

Key words: grasp force; movement precision

\section{Significance Statement}

Humans use their hands on a daily basis to interact with the environment. Many tasks require the hand's movement to be precise. Standard measures of movement precision resort to measuring the stiffness of the arm, which is notoriously difficult to measure during motion. We show that the power grasp force is correlated with movement precision, and that it provides a real-time measure of movement precision adaptation. Furthermore, the grasp force measure reveals that the brain has a state-dependent adaptation of movement precision, such that it increased grasped force in locations where the hand's deviation was greatest.

\section{Introduction}

Grasping with the hand is a fundamental motor action in humans that can be evoked in infants (McGraw, 1940), alongside the traction response, where the passive stretching of the shoulder abductors and the arm's flexors cause the fingers, elbow, and shoulder flexors to flex in synergistic response (Twitchell, 1965). As infants mature, their arm movements become smoother and more precise (Thelen et al., 1996). As adults, humans rarely make mis-

Received March 13, 2019; accepted June 21, 2019; First published July 11, 2019.

The authors declare no competing financial interests.

Author contributions: A.T., H.K., and Y.K. designed research; A.T. performed research; A.T. analyzed data; A.T., H.K., and Y.K. wrote the paper. takes when moving the arm, like when reaching to grab a mug. However, some skilled movements that require precision are difficult even for adults.

Taking hammering as an example, the hammer must strike the nail head precisely, which is challenging due to self-generated motor noise (Selen et al., 2009). The head of the hammer must remain flat against the nail during contact, which is difficult as unpredictable contact forces can destabilize the hammer (Ganesh et al., 2012). Failure

A.T. was partially supported by the Japan Science and Technology Agency (JST) Precursory Research for Embryonic Science and Technology Grant JPMJPR18J5 and Japan Society for the Promotion of Science (JSPS) Grantsin-Aid for Scientific Research (KAKENHI) Grant JP18K18130. A.T., H.K., and Y.K. were partially supported by the JST Mirai Grant JPMJMI18C8. 
at such tasks occurs when the hand's movement is perturbed unpredictably because the central nervous system (CNS) uses delayed sensory feedback to correct its movement (Pew et al., 1967). Thus, both unpredictable selfgenerated motor noise and environmental interactions result in reduced movement precision that cannot be corrected immediately by the CNS. It should be noted that precision is different from accuracy, as precision relates to variance whereas accuracy refers to bias.

A vast literature exists on how humans adapt to a force field that perturbs the accuracy of the hand's motion when reaching toward a point target (Shadmehr et al., 2010). The pioneering study of Shadmehr and MussaIvaldi (1994) revealed the ability of the CNS to learn to move in a velocity-dependent force field. Before the introduction of the force field, the hand moves from one point to another in a straight but somewhat curved trajectory. When the force field is introduced, the hand's trajectory curves outward and causes the subject to miss the intended target. Movement accuracy is regained by learning the dynamics of the force field and countering the force field's effects on the hand via appropriate forces produced by the hand (Conditt et al., 1997).

To recover movement accuracy in the force field, a forward model of the force field's dynamics is learned by the CNS. However, if the external forces are unpredictable and cause movement imprecision, the CNS uses a different strategy of coactivating the muscle pairs in the arm to increase its stiffness, which reduces the impact of unpredictable forces on the movement of the arm (Hogan, 1984; Burdet et al., 2001; Wong et al., 2009). Thus, the CNS' adaptation of movement precision in the presence of unpredictable external forces has been estimated by measuring the stiffness or the cocontraction of the arm (Franklin et al., 2008).

A recent study reported another method of measuring the CNS' adaptation to unpredictable forces. The authors of this study measured increases in the pinch grip force when subjects reached in an unpredictable force field (Hadjiosif and Smith, 2015). They found a positive correlation between the pinch grip force and the variability of the external forces. One issue with the pinch grip methodology is the strong coupling between the pinch grip force and the load force, which may confound the interpretation of the data. Furthermore, Hadjiosif and Smith (2015) did not test whether subjects increase pinch grip force when increased movement precision is desired in the absence of external forces.

In this study, we hypothesize that the power grasp force positively correlates with the desire to increase movement precision. We test two predictions based on this hypothesis in two experiments. First, we test the hypothesis that the grasp force increases when subjects want to improve

Correspondence should be addressed to A. Takagi at takagi.a.ae@m.titech.ac.jp. https://doi.org/10.1523/ENEURO.0095-19.2019

Copyright (C) 2019 Takagi et al.

This is an open-access article distributed under the terms of the Creative Commons Attribution 4.0 International license, which permits unrestricted use, distribution and reproduction in any medium provided that the original work is properly attributed. movement precision. This hypothesis is tested by asking subjects to keep their hand within a wide or narrow visual track while reaching toward a target, and measuring the changes in the grasp force. Second, we hypothesize that changes in grasp force reflect a desire to improve movement precision, and do not reflect the actual movement precision per se. This second hypothesis is tested by asking subjects to reach in a diverging force field that pushed their hand laterally from the center line. As subjects were unaware of when the force field would activate, we predicted that the grasp force would not increase in response to the force field on the first trial. We also predicted that in catch trials, where the force field was unexpectedly turned off, the grasp force would not decrease although the actual movement precision was high.

\section{Materials and Methods}

Ten male subjects, who all gave their informed consent, participated in the study. The experiment was conducted in accordance with the Declaration of Helsinki, and the study was approved by the Ethical Review Board for epidemiological Studies at the Tokyo Institute of Technology.

The subjects were seated facing the KINARM planar robotic manipulandum from BKIN Technologies (Fig. 1). Subjects held onto the KINARM interface via a handle that was affixed with a three-axis force sensor (Tec Gihan) to measure the grasp force from the palm of the hand during reaching movements. An Edero Armon arm support was used under the elbow to support the arm's weight when using the robotic interface. Visual feedback was provided on a monitor that was placed upside-down such that subjects viewed a reflection of the monitor on a thin film mirror placed above the hand, which obscured it from view. The position of the hand was visible as a white circular cursor during both the visual track and the divergent force field experiments.

\section{Visual track reaching experiment}

Subjects moved their hand to reach a target of radius 2 $\mathrm{cm}$ placed $25 \mathrm{~cm}$ away from their initial starting position. Subjects were instructed to prevent the cursor from deviating off a red visual track that was displayed between the initial and target positions (Fig. 1B).

The experimental structure consisted of seven blocks where each block contained 15 trials. The first block consisted of training trials, where the red region was absent. Blocks 2, 4, and 6 were the "wide" condition that demanded low movement accuracy blocks as the visual track was $\pm 4 \mathrm{~cm}$ wide. On the other hand, the "narrow" condition tested in blocks 3,5 , and 7 required high movement accuracy due to a narrow visual track with a width of $\pm 0.4 \mathrm{~cm}$. The cursor in this experiment had a diameter of $0.4 \mathrm{~cm}$. Feedback of the movement duration was provided to the subject on a trial-by-trial basis. Movements that were faster than $900 \mathrm{~ms}$ and longer than $1100 \mathrm{~ms}$ triggered a feedback message of "fast" or "slow," respectively, to ensure that subjects reached with comparable movement speeds in both the wide and narrow conditions. No feedback was given of the lateral deviation after the trial. 
A

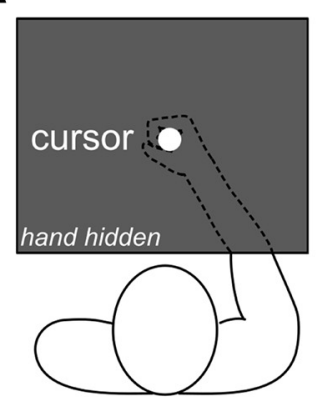

robot

handle

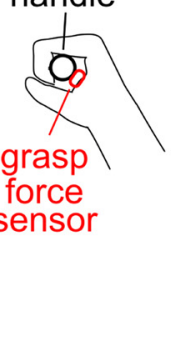

B

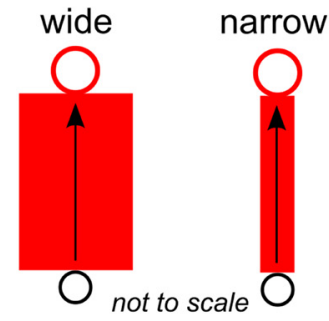

Experiment 1

reaching along visual track

C

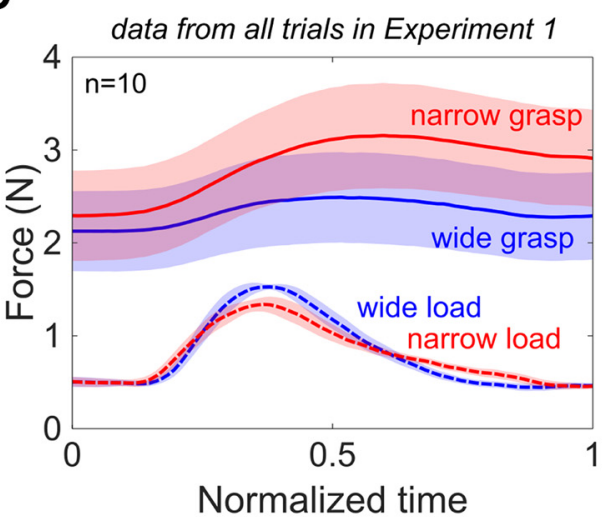

D
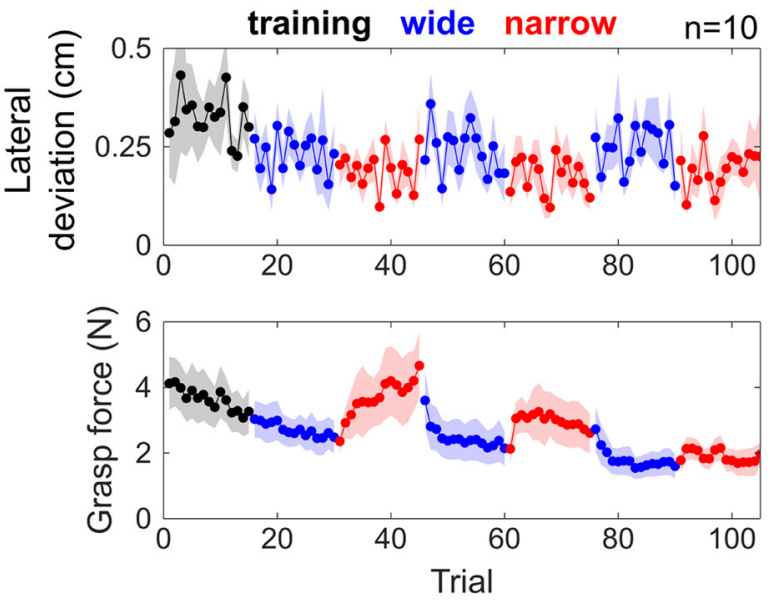

E

$n=10$
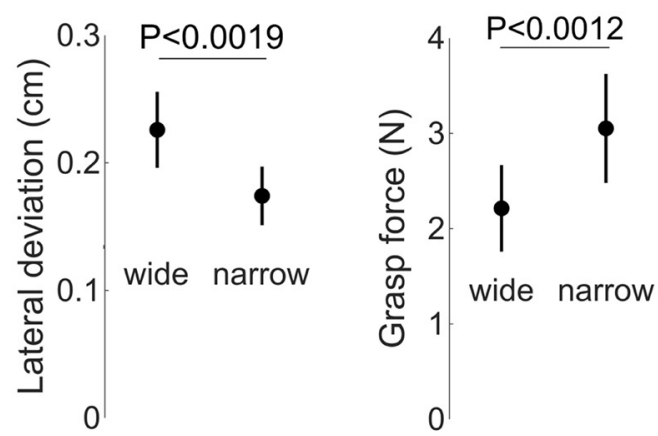

F

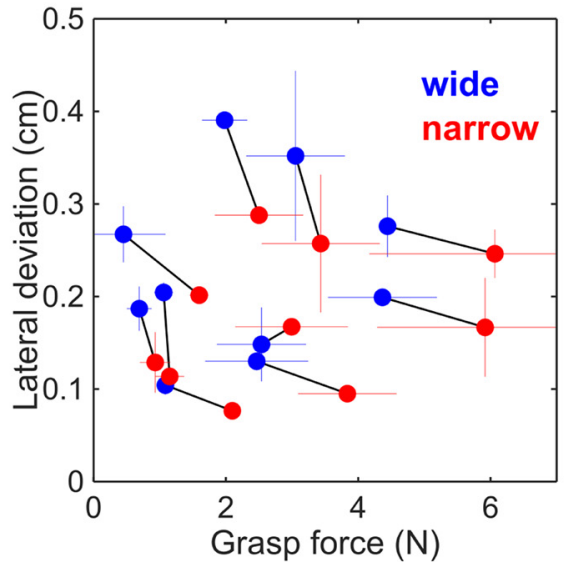

Figure 1. Experimental setup, protocol, and results from the first experiment where subjects reached along a visual track $\boldsymbol{A}$, Subjects held the handle of a robotic manipulandum, where the position of their arm was hidden behind a film mirror. Subjects received visual feedback of the position of their hand as a cursor on the monitor such that it could be seen above the hand. The elbow was supported such that the hand, elbow, and shoulder were level along a horizontal plane. A force sensor was placed between the palm of the hand and the handle to measure the grasp force. $\boldsymbol{B}$, In the first experiment of reaching along a visual track, subjects were presented with two visual feedback conditions showing either a wide or a narrow track. Subjects were instructed to keep their cursor inside the track and reach the circular target at the end of the track. Subjects first made reaching movements without a visual track in a training block, after which they experienced wide and narrow blocks in consecutive sequence for three repetitions per condition. $\boldsymbol{C}$, The group mean grasp force (solid traces) and load force (dotted traces) are plotted as a function of normalized time, where the shaded area is 1 SE. The data were separated into the wide (blue) and the narrow (red) conditions. The Pearson correlation coefficient between the grasp and load forces was not significantly different from zero. Thus, no significant correlation between grasp and load force was observed when reaching along a visual track. $\boldsymbol{D}$. The lateral deviation and the grasp force of the population mean and SE are plotted as a function of trials with the plot color indicating the training trials (black), wide track (blue) and narrow track (red) conditions. $\boldsymbol{E}$, The mean lateral deviation and the mean grasp force from the population is shown for the wide and narrow conditions. The lateral deviation was lower and the grasp force was higher when the visual track was narrow. $\boldsymbol{F}$, The lateral deviation is plotted as a function of the grasp for every subject in the wide and narrow conditions, with a black line connecting the data from the same subject. The thin blue and red lines show standard deviation from the linear mixed effects model fits. An increase in grasp force was observed to subsequently reduce the lateral deviation. 
As we observed a linear relationship between both the lateral deviation and the grasp force as a function of trials, we fitted these data from the visual track experiment using the linear mixed-effects model of the form

$$
Y=\beta_{0 s}+\beta_{1 s} C+\beta_{2 s} T+\beta_{3 s}(T \cdot C)+\varepsilon_{s},
$$

where the response $Y$ is either the vector of data from grasp force $F_{G}$ or from the lateral deviation $x_{L D}, T$ is the trial number, $C$ is the visual track condition (narrow or wide), $\beta_{0 s}$ is the intercept, $\beta_{1 s}$ to $\beta_{3 s}$ are the parameters for each predictor, and $\varepsilon_{s}$ is the unexplained variance of the response $Y$ for each subject $s$.

A likelihood ratio test was employed to examine the significance of the condition parameter $C$ on explaining the data. If deemed significant, this implied that the width of the visual track had a significant impact on the grasp force and the lateral deviation.

If the condition was deemed to significantly influence the grasp force or the lateral deviation, a one-sample $t$ test was conducted on the data, which was grouped separately for the wide and narrow conditions. This enabled us to test our hypothesis of whether the grasp force increased when reaching along a narrow visual track and whether a reduction in lateral deviation was observed in the narrow track. However, these tests alone were not sufficient to establish a relationship between the grasp force and the lateral deviation as they only examine the effect on a group level. We examined how each subject's lateral deviation changed as a function of the change in their grasp force. Here, a non-parametric sign test was employed as these data were observed to violate normality. The normality of all datasets was tested using an Anderson-Darling test before post hoc testing.

\section{Divergent force field experiment}

The same 10 subjects that took part in the first experiment participated in the divergent force field task (Fig. $2 A)$. Subjects were instructed to reach a target $20 \mathrm{~cm}$ away from the starting position. The diameter of the cursor was $1 \mathrm{~cm}$ in this experiment. Feedback was provided on a trial-by-trial basis about the duration of the movement, which had to be between 500 and 600 ms. Each participant practiced a standard reaching task for 15 training trials, after which they experienced 25 divergent field blocks. The divergent force field was designed to amplify lateral reaching errors by applying the following force to the hand,

$$
F_{x}=k x \text { for }|x|<0.04 m,
$$

where the stiffness $k=400 \mathrm{~N} / \mathrm{m}$ and $x$ is the lateral position of the hand such that the initial and target positions are at $x=0$. Each divergent field block was composed of four trials where the last trial was a catch trial where the force field was switched off, i.e., $F_{x}=0$. The catch trials tested whether subjects were simply grasping the handle as a reaction to the forces from the robot, or were increasing their grasp force to improve their movement precision. If the hand's lateral deviation was greater than $\pm 4 \mathrm{~cm}$ in a force field trial, the force field was switched off and the subjects were shown a "failed" trial message.
Post hoc one-sample $t$ tests were conducted to examine the difference between the last training trial and the first force field trial, and the difference between the catch trials and the force field trials. If the grasp force is different in either of these cases, our hypothesis must be rejected.

\section{Results}

\section{Experiment 1: reaching along a wide or narrow visual track}

In the first experiment, subjects had to make point-topoint reaching movements toward a circular target of radius $2 \mathrm{~cm}$ that was placed $25 \mathrm{~cm}$ away from the initial starting position. Subjects were instructed to prevent the cursor from deviating off a red visual track that was displayed between the initial and target positions (Fig. 1B).

The literature reports that, with a pinch grip, significant correlation is observed between the pinch grip force and the load force (Flanagan and Wing, 1997). Such a correlation could undermine our study as the grasp force may simply reflect the load experienced by the hand during reaching. The grasp force and the load force are plotted as a function of normalized time in Figure 1C. The data from every trial's whole movement were selected for this analysis, where the start of the trial was when the target appeared, and the end was when the hand stopped inside the target. We calculated the Pearson correlation coefficient in all trials between the grasp force and the load force supplied by the subject against the robotic interface. If the load force has a significant impact on the grasp force measurement, this must be taken into account in subsequent analysis as it may influence the results. However, we found that the group mean correlation between the grasp and load force was $\rho=0.26 \pm 0.12$ (mean and $\mathrm{SE}$ ), which was not significantly different from zero (onesample $t$ test, $t_{(9)}=2.09, p>0.07$ ). Thus, no significant coupling was observed between the grasp force and load force in this experiment.

Next, we examined whether the variance in the reaching movement was different between the wide and narrow conditions. We normalized all trajectories in time and calculated the mean trajectory for each wide and narrow condition using the trials from all three blocks. We then calculated the lateral deviation, defined as the absolute distance halfway into the movement between the cursor's $x$-axis position and the mean trajectory, for each trial, which is plotted as a function of trials for the population mean in Figure $1 D$. The lateral deviation appeared to be functionally dependent on the width of the visual track. We employed a fit with a linear mixed-effects model (Eq. 1) on the data from all trials, which was labeled by subject, trial number and track condition. A likelihood ratio test revealed a significant effect of the visual track condition on the lateral deviation $\left(\chi^{2}(2)=21.95, p<10^{-4}\right)$. Using the regressed linear model, we calculated the difference in the lateral deviation between the wide and narrow track conditions for the trial range 16-115, i.e., blocks 2-7. A one-sample $t$ test on the lateral deviation showed that the subjects' mean lateral deviation, shown in Figure $1 E$, was significantly smaller in the narrow $(0.17 \pm 0.02 \mathrm{~cm})$ than 
A

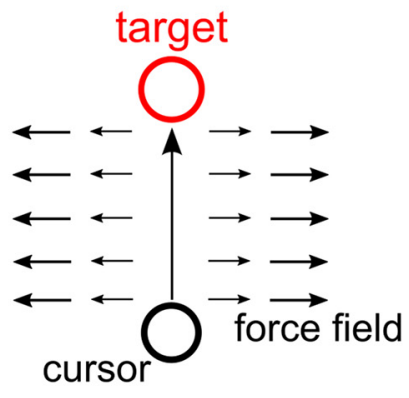

B

first 15 trajectories

in DF from sample subject

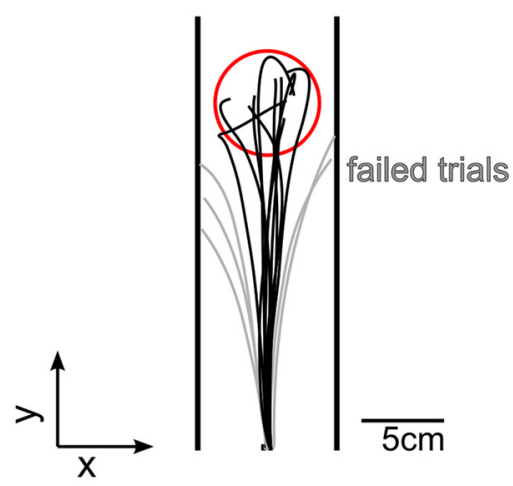

Experiment 2

reaching in a divergent force field

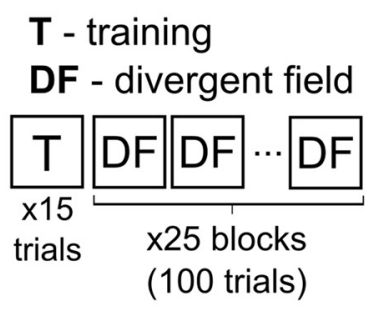

C

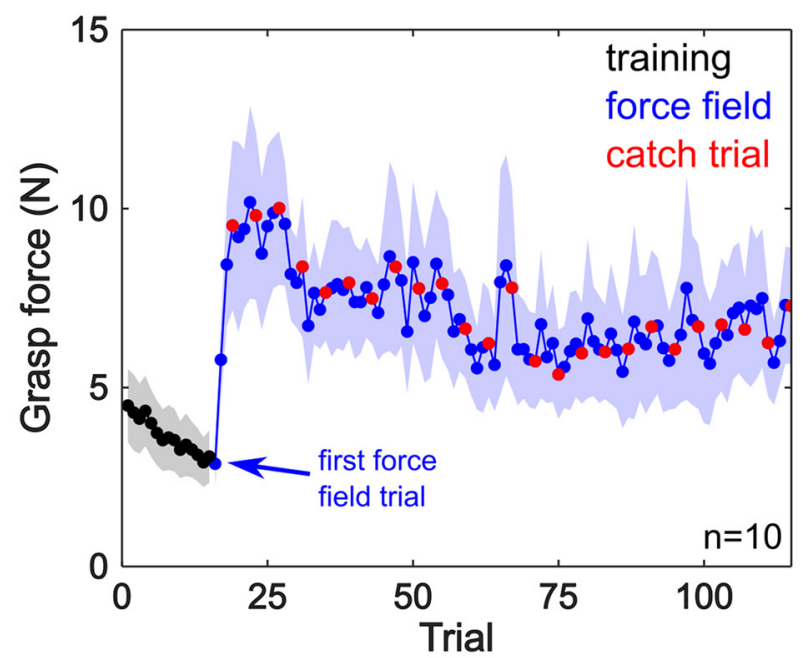

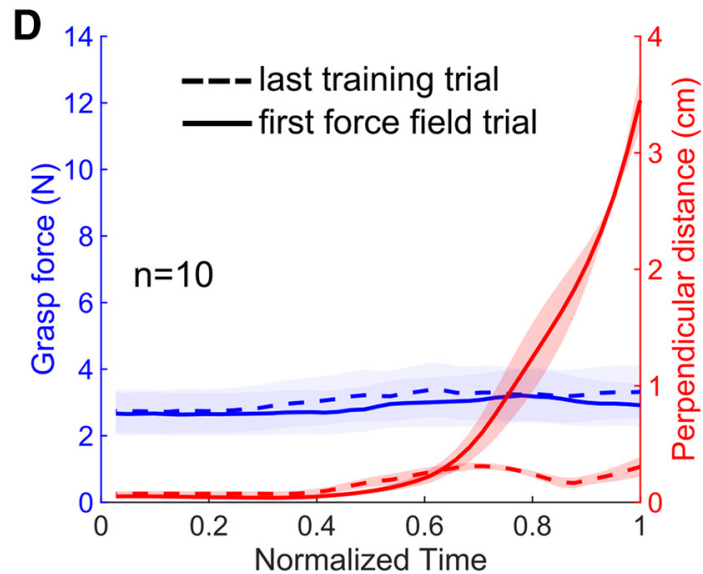

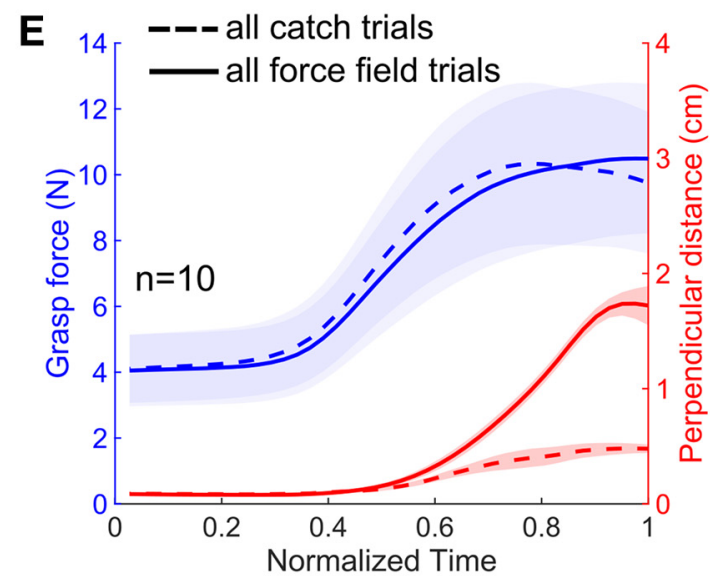

Figure 2. The divergent force field reaching experiment supports the hypothesis that the grasp force increases when better movement precision is desired, and not when the actual movement precision changes. $\boldsymbol{A}$, A schematic of the experiment and its protocol. The force field pushed the hand away laterally if it deviated from the line that connected the start and the target positions. To succeed, a subject must reach as straight as possible with minimum lateral deviation. Subjects experienced 15 training trials in the null field conditions, after which they experienced 100 force field trials. Of these 100 trials, every fourth trial was a catch trial where the force field was switched off. $\boldsymbol{B}$, The first 15 trajectories in the force field trials from a sample subject are shown, where the gray trajectories show failed trials where their hand hit the safety margins placed $4 \mathrm{~cm}$ to the left and right of the center line. A trial was successful when subjects stopped inside the red target. $\boldsymbol{C}$, The mean grasp force from all subjects, averaged over each trial, is plotted as a function of trials. In training trials (black), the grasp force continually declines. The grasp force in the first force field trial is similar to the level observed in training trials, but begins to increase until it peaks at approximately the fourth force field trial. Although the grasp force declines during the force field trials, it never reaches the same level as the training trials. Furthermore, the grasp force in catch trials is indistinguishable from the force field trials, revealing that the grasp is not a reaction to the forces from the force field, but is premeditated. $\boldsymbol{D}$, The group mean grasp force from the last training trial (dashed blue trace) and the first force field trial (solid blue trace) are plotted as a function of normalized time. On the same figure, the group mean perpendicular distance from the last training trial (dashed red trace) and the first force field trial (solid red trace) are plotted as a function of normalized time. Although the 
continued

perpendicular distance increased dramatically due to the force field, the grasp force remained constant. $\boldsymbol{E}$, The group mean grasp force (blue traces) and the group mean perpendicular distance (red traces) from all catch trials (dashed blue trace) and all force field trials (solid blue trace) are plotted as a function of normalized time. The grasp force was similar between the catch trials and the force field trials, although the perpendicular distance was smaller in catch trials where the force field was switched off.

the wide $(0.23 \pm 0.03 \mathrm{~cm})$ condition $\left(t_{(9)}=-4.32, p<\right.$ $0.0019)$, indicating that the subjects' trajectories were more precise in the narrow condition. What facilitated the subjects' ability to improve their lateral movement precision in the narrow track?

If the grasp force is correlated with movement precision during reaching, a selective increase in grasp force should be observed in the narrow condition where smaller lateral deviation was observed. The population mean grasp force from each trial is plotted as a function of trials in Figure $1 B$, where the blue and red points are from the wide and narrow conditions, respectively. The average grasp force from a single trial was calculated using data from the entire movement, where the start of the trial was when the target appeared, and the end of the trial was when the hand stopped inside the target. On visual inspection, there appeared to be a functional dependence of the grasp force on the visual track condition and the trial number. The grasp force data from all trials and all subjects were fit with a linear mixed-effects model with the trial number and track condition as predictors (refer to Eq 1). A likelihood ratio test showed that the grasp force was significantly affected by the visual track condition $\left(\chi^{2}(2)=\right.$ $118, p<10^{-15}$ ). We calculated the mean grasp force in the wide and narrow conditions from the linear model fits, and a one-sample $t$ test showed that the grasp force was significantly higher in the narrow than in the wide condition $\left(t_{(9)}=4.41, p<0.0012\right)$.

To directly assess the effect of the grasp force on the movement, we plotted the lateral deviation as a function of the grasp force for each subject in the wide (blue) and narrow (red) conditions in Figure $1 F$, with a black line connecting the data from the same subject. Data from each subject were averaged across all three blocks in the wide and narrow conditions to yield one data point per subject per condition. An increase in grasp force was observed to reduce the hand's lateral deviation, and a non-parametric sign test, which was employed since the data violated normality according to an Anderson-Darling test, found this relationship to be significant $(p<0.022$ ). In summary, these results suggest that the grasp force is related to the hand's movement precision during reaching.

\section{Experiment 2: divergent force field}

In the second experiment, we tested subjects reaching in a divergent force field. The force field applied a force that pushed the hand laterally away from the center line if it deviated laterally from the straight line between the initial and target positions (Fig. 2A). The same 10 subjects that took part in the first experiment participated in the divergent force field task (Fig. 2A). The trajectories from the first 15 trials inside the divergent force field from a sample subject are plotted in Figure 2B. The grasp force, averaged over each trial, is plotted as a function of trials in Figure $2 C$, where the points are the group mean and the shaded area is the SE from all 10 subjects. In the 15 training trials, where subjects reached toward the target without the force field, the grasp force was generally low (Fig. 2C, black dots) and continually declined with practice. On trial 16, when the force field was first experienced by subjects, the grasp force was effectively the same as the last training trial. The grasp force increased rapidly in the second and third force field trials, and peaked at approximately the fourth force field trial, after which the grasp force declined exponentially but not to the original level observed in the training trials.

Recall that the second prediction from our hypothesis dictates that increases in the grasp force should only be related to a desired increase in movement precision. Thus, the grasp force should not change even if the actual movement precision increases or decreases. The movement precision in this force field experiment is denoted by the perpendicular distance, defined as the absolute distance from the line at $x=0$.

First, we examined how the grasp force and the perpendicular distance changed from the last training trial (Fig. 2D, dashed trace) to the first force field trial (solid trace). The group mean grasp force and the group mean perpendicular distance are plotted, in Figure $2 D$, as a function of normalized time, where time 0 was the time of target onset and the end was where the subject reached the target. The perpendicular distance was observed to increase dramatically on the first force field trial. The group mean grasp force in the last training trial was $3.1 \pm$ $0.7 \mathrm{~N}$ (mean and SE) and for the first force field trial it was $2.9 \pm 0.7 \mathrm{~N}$. A paired sample $t$ test found that the difference in the grasp force between the last training trial and the first force field trial was not statistically significant $\left(t_{(9)}\right.$ $=2.20, p>0.055)$. Although the perpendicular distance increased dramatically, the grasp force did not change.

Next, we examined whether the grasp force was different on catch trials in comparison to the force field trials. The group mean grasp force (blue traces) and the perpendicular distance (dashed traces) from all catch trials (dashed traces) and all force field trials (solid traces) is plotted as a function of normalized time. Notably, the grasp force profile is different from the training trial in Figure 2D, where it was constant throughout the movement. The grasp force appears to increase in tandem with the perpendicular distance in the force field trials. We found the mean grasp force, taken over the whole trial, and calculated the difference between the mean grasp force in catch trials with the neighboring force field trials. This difference was $0.08 \pm 0.12 \mathrm{~N}$, which was statistically not different from zero (one-sample $t$ test, $t_{(9)}=0.65, p>$ 0.53). Hence, the grasp force did not change in catch 
trials, although the perpendicular distance clearly decreased.

\section{Discussion}

In this study, we measured the power grasp force while subjects completed two reaching tasks. The first task asked subjects to stay within a visual track during reaching, and the second task had subjects reach to a target while their hand was perturbed by a diverging force field that amplified lateral reaching errors. The results from both experiments support our hypothesis that the grasp force is positively correlated with the desire to increase movement precision. Namely, the grasp force increased when the visual track was narrower and required higher movement precision. Furthermore, the grasp force in the force field did not change in tandem with changes in the actual perpendicular distance, but with the desire to change it.

The latter observation, that the grasp force was not significantly correlated with the load force from the force field, is of importance. Several studies have reported the high correlation between the pinch grip force and the load force from the environment (Flanagan and Wing, 1997; Flanagan et al., 2006). This coupling had to be taken into account when subjects adapted their pinch grip force when reaching in a variable force field (Hadjiosif and Smith, 2015) to avoid the confound where a change in the grip force may be mistaken for an adaptation to the variability of the force field. Instead, the change may have been due to the increased load force from the variable force field. In our experiment, we employed a divergent force field, which has a similar effect to the variable force field used in Hadjiosif and Smith (2015), namely that unpredictable forces are imposed on the subject's hand that cause movement variability. Unlike the pinch grip force, the power grasp force during reaching was not correlated with the load force from the force field. The power grasp force thus avoids confounds when interpreting changes in the grasp force. However, this may be valid only when the forces from the force field are approximately orthogonal to the placement of the grasp force sensor, and so caution is still required when interpreting the changes in the grasp force.

In both experiments, the grasp force increased from the initial exposure to a visual or force field condition, but gradually decayed as a function of trials. As reported in Hadjiosif and Smith (2015), two opposing adaptation phenomena are likely at play. The first is a fast and sensitive adaptation that increases grasp force to rapidly improve movement precision due to a task demand. The second is a slower adaptation process that optimizes grasp force to conserve effort (Todorov and Jordan, 2002; Emken et al., 2007). The summed contributions of both fast and slow adaptation processes explain why the grasp force increases rapidly from initial exposure, but continually decays throughout our experiment. This decay can be observed in the training trials in both experiments, implying that the gradual decay in the grasp force must be taken into account when interpreting the data. In our first experiment, this was accounted for by the linear mixedeffects model that included a trial gradient. The decay in the grasp force does not contradict our hypothesis, but it shows that an effort conservation process is continually working to reduce excessive grasp force production.

In addition to these adaptation processes, we observed that the increase in grasp force, when switching from a wide to a narrow visual track, was less pronounced in the later trials. Subjects may have learned to update their motion plan to move straighter without having to rely on increasing grasp force to remain inside the visual track (Wong et al., 2009). Such a strategy was likely possible when staying inside the narrow visual track but was infeasible when reaching in the diverging force field that punished even minor lateral deviations. This may explain why the grasp force plateaus to a value above the nominal level observed in the training trials in the divergent force field experiment.

The changes in the grasp force observed when subjects learned to reach in the diverging force field are similar to the results of another study that examined the adaptation of arm cocontraction during the learning of a divergent force field (Franklin et al., 2008). In their study, they also found an initial, rapid increase in cocontraction, followed by a slow and gradual decay, which plateaued above the baseline observed in the training trials. The similarity between the grasp force observed in our study, and the arm cocontraction observed in the study of Franklin et al. (2008), raises the possibility of using the grasp force as a tool to probe the CNS' desire to improve movement precision in rapid movements, such as during a golf swing (Komi et al., 2008), where the delay introduced by the processing of the electromyography data may be detrimental to the analysis. As such, the grasp force methodology could complement existing methods to measure the cocontraction of muscles to further our understanding of the CNS' desire to improve movement precision.

\section{References}

Burdet E, Osu R, Franklin DW, Milner TE, Kawato M (2001) The central nervous system stabilizes unstable dynamics by learning optimal impedance. Nature 414:446-449.

Conditt MA, Gandolfo F, Mussa-Ivaldi FA (1997) The motor system does not learn the dynamics of the arm by rote memorization of past experience. J Neurophysiol 78:554-560.

Emken JL, Benitez R, Sideris A, Bobrow JE, Reinkensmeyer DJ (2007) Motor adaptation as a greedy optimization of error and effort. J Neurophysiol 97:3997-4006.

Flanagan JR, Wing AM (1997) The role of internal models in motion planning and control: evidence from grip force adjustments during movements of hand-held loads. J Neurosci 17:1519-1528.

Flanagan JR, Bowman MC, Johansson RS (2006) Control strategies in object manipulation tasks. Curr Opin Neurobiol 16:650-659.

Franklin DW, Burdet E, Tee KP, Osu R, Chew C-M, Milner TE, Kawato M (2008) CNS learns stable, accurate, and efficient movements using a simple algorithm. J Neurosci 28:11165-11173.

Ganesh G, Jarrasse N, Haddadin S, Albu-Schaeffer A, Burdet E (2012) A versatile biomimetic controller for contact tooling and haptic exploration. Presented at the 2012 IEEE International Conference on Robotics and Automation (ICRA), pp 3329-3334. Saint Paul, MN:IEEE.

Hadjiosif AM, Smith MA (2015) Flexible control of safety margins for action based on environmental variability. J Neurosci 35:91069121. 
Hogan N (1984) Adaptive control of mechanical impedance by coactivation of antagonist muscles. IEEE Trans Autom Control 29:681-690.

Komi ER, Roberts JR, Rothberg SJ (2008) Measurement and analysis of grip force during a golf shot. Proc Inst Mech Eng Part P J Sports Eng Technol 222:23-35.

McGraw MB (1940) Suspension grasp behavior of the human infant. Am J Dis Child 60:799-811.

Pew RW, Duffendack JC, Fensch LK (1967) Sine-wave tracking revisited. IEEE Trans Hum Factors Electron HFE-8:130-134.

Selen LPJ, Franklin DW, Wolpert DM (2009) Impedance control reduces instability that arises from motor noise. J Neurosci 29: 12606-12616.

Shadmehr R, Mussa-Ivaldi FA (1994) Adaptive representation of dynamics during learning of a motor task. J Neurosci 14:3208-3224.
Shadmehr R, Smith MA, Krakauer JW (2010) Error correction, sensory prediction, and adaptation in motor control. Annu Rev Neurosci 33:89-108.

Thelen E, Corbetta D, Spencer JP (1996) Development of reaching during the first year: role of movement speed. J Exp Psychol Hum Percept Perform 22:1059-1076.

Todorov E, Jordan MI (2002) Optimal feedback control as a theory of motor coordination. Nat Neurosci 5:1226-1235.

Twitchell TE (1965) The automatic grasping responses of infants. Neuropsychologia 3:247-259.

Wong J, Wilson ET, Malfait N, Gribble PL (2009) Limb stiffness is modulated with spatial accuracy requirements during movement in the absence of destabilizing forces. J Neurophysiol 101:1542-1549. 